\title{
TSR method for burns investigation approach
}

by M. Kaczmarek*

\author{
*Gdansk Univ. of Technology, 80-233, Narutowicza Str., Gdansk, Poland, markaczm@pg.edu.pl
}

\begin{abstract}
The article presents the possibilities of applying the TSR method for the diagnosis of burn wounds. The wound area is stimulated by cold gas and a sequence of thermograms of heating the tissue is recorded. Next, TSR algorithms are used to determine the parameters that allow the wound to be classified into one of the classes: the wound heals within three weeks, the wound will not heal in three weeks. The method was validated on a series of in-vivo experiments on animals and on several clinical cases.
\end{abstract}

\section{Introduction}

The research teams at the Biomedical Engineering Department of Gdansk University of Technology and in the Plastic Surgery Department of Gdansk Medical University have been working together in the field of IR-thermal imaging for the evaluation of burn wound depths for many years [1][2]. Previous publications by this group have evaluated the usefulness of static thermography (ST) and active dynamic thermography (ADT). Detailed analysis of the heat transfer processes in burned and healthy tissues as well as analysis of heat exchange conditions within the environment allowed for further improvements to the ADT method. In particular, the use of the cold excitation allows for more precise evaluation of the depth of burn wounds. Firstly, the measurable signal may have a higher signal to noise ratio (SNR); secondly, the conditions of heat exchange and the interpretation of measurement data is, in this case straightforward and much less dependent on external temperature.

The thermographic signal reconstruction (TSR) method is recently, in addition to pulsed phase thermography (PPT) and differential absolute contrast, lock-in or active dynamic thermography (ADT), one of the most popular image processing techniques in excited thermography [3][4][5].

Reconstruction of a signal usually means its adaptation to a certain model. In thermographic studies, this process is carried out by approximating the temperature dynamic changes of the tested object in time. In the TSR method, the temperature flux of each thermogram pixel is replaced by a polynomial approximation determined using the least squares method, which allows a good quality to match the course of the temperature history of the examined surface, and not to sudden noise fluctuations. The reconstruction process leads to the separation of temporal and spatial noise components in a series of images and allows for a significant reduction of time noise.

The TSR method, originally developed as a research technique to detect defects in materials in pulsed thermography experiments, is based on the assumption that the temperature profiles obtained from the thermographic sequence recorded after the thermal pulse, of the pixels without defects should be consistent with the one-dimensional curve of the Fourier equation describing the propagation of the thermal wave in a homogeneous material that can be written in a logarithmic form as:

$$
\ln (T)=\ln \left(\frac{Q}{e}\right)-\frac{1}{2} \ln (\pi t)
$$

where: T-temperature of the selected pixel at time $t, \mathrm{Q}$ - thermal pulse energy, $e$ - thermal effusivity of the medium. In our approach we try to fit the measured data to logarithmic polynomial as in equation (2):

$$
\ln (T)=a_{0}+a_{1} \ln (t)+a_{2}[\ln (t)]^{2}+\ldots+a_{n}[\ln (t)]^{n},
$$

wherein the temperature $\mathrm{T}$ is a function of the time $t$ for each pixel of the image with spatial coordinates $(i, j)$.

In this paper, thermal excitation in the form of cooling stimulus, was applied by switching on and off a set of cryogenic devices, a stream of a dry mixture of $\mathrm{CO}_{2}$ and ambient air was used. A series of IR thermal images of a tested surface allowed for further analysis of the heat transfer processes allowing the thermo-physical material properties (thermal diffusivity and conductivity) to be quantified is analysed using the TSR approach with $1^{\text {st }}$ and $2^{\text {nd }}$ derivations calculation.

It should be underlined that medical applications of QIRT comparing to technical NDE are much more difficult for interpretation as heat transfers in living tissues are far more complicated comparing to technical applications. First, the human body is a very complicated structure, alive and geometrically flexible; second, thermal boundary conditions are usually not fully known and what is more, temperature distribution inside a tested structure usually is not uniform. 
Additionally natural biofeedback dynamic reactions of living organisms and tissues may be strong and should be avoided to get clear interpretation of measurement results. This general heat transfer model does not include convection neither heat delivered by blood perfusion. Therefore temperature distribution and its change in time during and after excitation in biological tissue are described by the bio-heat transfer equation proposed by Pennes [10]:

$$
c_{t} \rho_{t} \frac{\partial T(x, y, z, t)}{\partial t}=k \nabla^{2} T(x, y, z, t)+Q_{b}+Q_{m}+Q_{z}
$$

where: $k \nabla^{2} T(x, y, z, t)$ - volumetric power density exchanged due to temperature gradient and thermal conduction, $Q_{b}$ - volumetric power density exchanged due to blood perfusion, $Q_{m}$ - volumetric power density caused by metabolism, $Q_{z}$ - volumetric dissipated power density of an external heating source.

When one analyse the heat flow in composites or other artificial materials there is no $Q_{b}$ and $Q_{m}$ components. So the heat exchange processes are more predictable than in the living tissues.

\section{Materials and method}

The examination is usually conducted on the $2^{\text {nd }}$ post-burn day and all required conditions for ST and ADT thermography should be fulfilled. The thermographic IR camera FLIR A320G of thermal resolution 0.08K and spatial resolution 320x240 pixels was used. First the data of classical static thermography ST was collected, followed by the ADT and TSR procedures. The cooling excitation by the stream of a cold mixture of $\mathrm{CO}_{2}$ and dry air generated by the CryoFlex-T cryotherapy device (Metrum Cryoflex, UK) was of the sufficient efficiency for getting the final skin temperature to the ambient temperature. Material is more than 60 burn wounds induced under controlled conditions in invivo experiments on animals and several clinical cases of burns.

The results of an investigation into human burn wounds by means of thermographic methods involving several patients treated in the Department of Plastic Surgery of the Medical University of Gdansk - GUMed - have been also presented (by permission of the Independent Bioethics Committee on Scientific Investigation of GUMed). Here the case of a 19 year old cook burned by boiling oil is shown as an example.
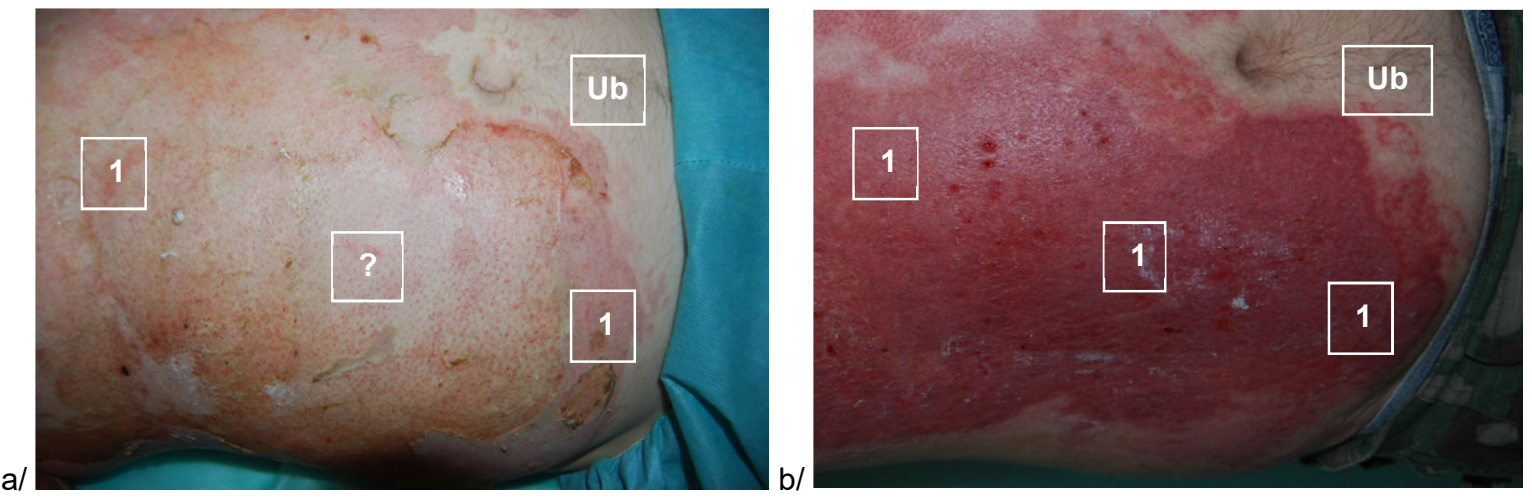

Fig. 1. Clinical application - a burn wound to the abdomen in 19 year old man - RGB photos taken a/ at the 2-nd day and b/ 21 days following burn accident

The histopathology of the biopsy specimen taken in the centre of the undetermined burn area was $50.4 \% \mathrm{dtms}$ (dermis thicknes in measure site). This result means that this part of the wound should heal within 3 weeks. Meaning of the indicated marks in Fig. 1:

1 - superficial burn, which will heal within 3 weeks,

? - undetermined burn wound,

Ub - unburned area (healthy skin).

The undetermined in visual inspection part of the wound was accurately diagnosed by means of the ADT method. The whole abdomen area was healed in 3 weeks.

\subsection{Temperature changes during natural tissue heating following active cooling}

For ADT analysis, the recorded temperature change is valuable following external stimulation stops. In the classic TSR method, the sample is excited with an ultra-short flash with the proper energy. In medical applications, a long thermal stimulus is used, and the observed temperature changes in a fairly long time. This implies specific 
discrepancies in the interpretation of results. In Fig. 2a the thermogram just following switch-off the excitation source (cooled area of the abdomen) is shown, in Fig. $2 \mathrm{~b}$ this same field of view following 60 seconds. The increase in temperature is noticeable but unevenly across the entire surface what is also evidenced on chart - Fig. $2 \mathrm{c}$ and $2 \mathrm{~d}$.

a)

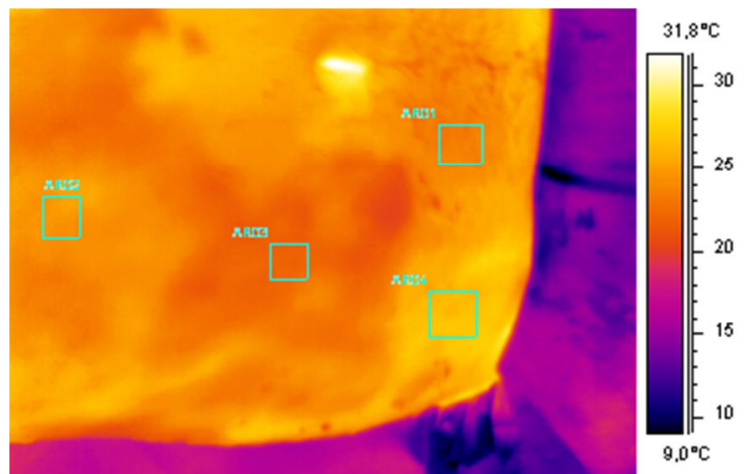

c)

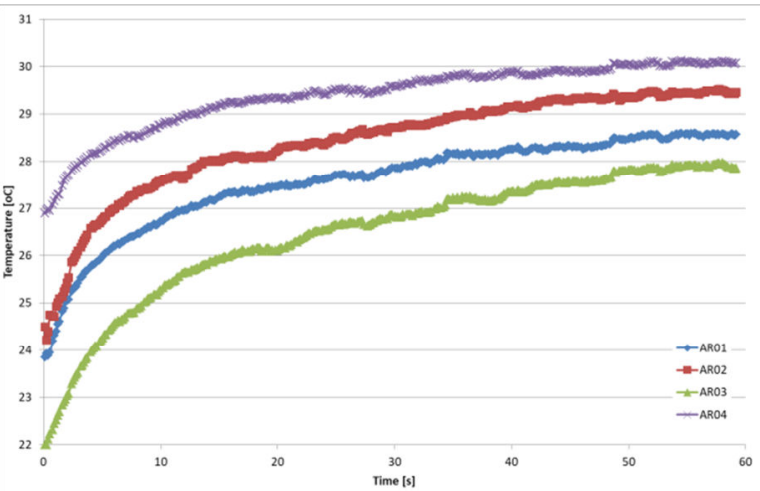
switch-off the excitation source at the 2-nd day, b) thermogram following next 60 second at the 2-nd day, c) temperature curves for region AR01-AR04 - linear scale, d) temperature curves for region AR01-AR04 - In-In (logarithmic) scale

As anyone can see in Figure 2c, it is very difficult to accurately determine the moment of switch-off of external stimulation. The excitation itself has the character of Gaussian curve, there are no sharp falling slopes. If we assume, for further analyses, the excitation switch-off time later by 1 second, the recorded waveforms of temperature in the logarithmic representation are linear in nature as shown in Figure $3 a$.

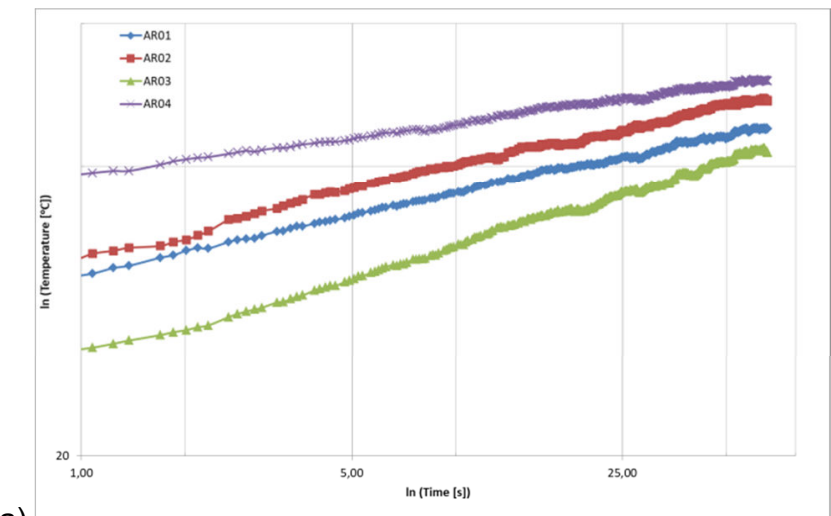

a)

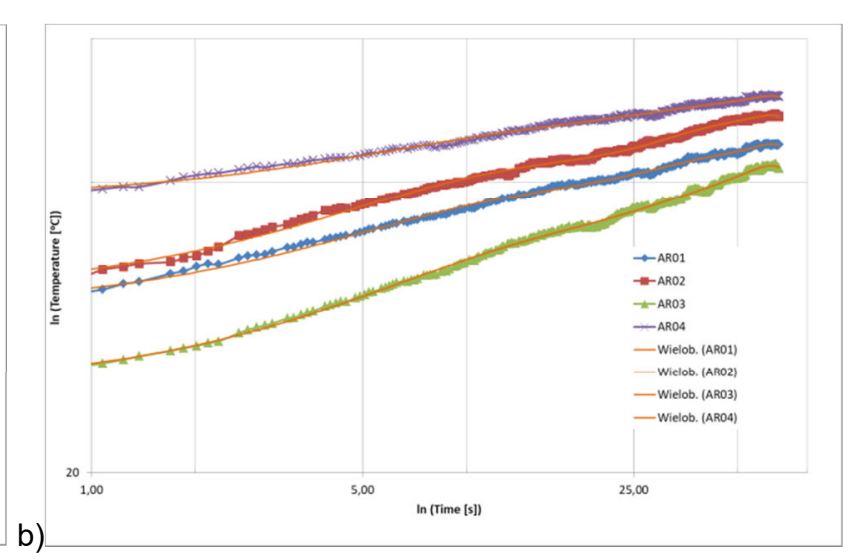

Fig. 3. Clinical application - a burn wound to the abdomen in 19 year old man; a) temperature curves for region AR01-AR04 - In-In (logarithmic) scale time of observation 1s-60s, b) fitted to polynomial model temperature curves for region AR01-AR04 - In-In (logarithmic) scale time of observation 1s-60s,

In Fig. 3b the temperature curves fitted to polynomial model for four regions of interest (AR01-AR04) according to Fig. 1 and Fig. $2 a$ are shown. The model is described by equation (2), where $n=6$. For lower polynomial degree the fitting is unsatisfactory and for higher polynomial degree it is difficult to interpret the physical meaning of the polynomial coefficients. 


\subsection{ADT methods with exponential model}

For ADT analysis, the recorded temperature change is valuable following external stimulation stops. Usually the twoexponential function is sufficient for practical applications, as accuracy of fitting measurement and model data is in this case almost perfect [1][2]. For pulsed cooling, at the natural recovery phase the appropriate function for each pixel is expressed by:

$$
T(x, y, t)=T_{\min }(x, y)+\Delta T_{1}(x, y)\left(1-e^{-t / \tau_{1}}(x, y)\right)++\Delta T_{2}(x, y)\left(1-e^{-t / \tau_{2}}(x, y)\right)
$$

where symbols here are: $T_{\min }=T_{\text {end_of_stimulus; }} \Delta T_{1}+\Delta T_{2}=\left(T_{0}-T_{\min }\right) ; \tau_{1}$ and $\tau_{2}$ respectively shorter and longer time constants; $\mathrm{T}_{0}$ - static temperature (before cooling).

Fortunately usually there are strong correlations between simple parametric model descriptors and theirs medical diagnostic values. It has to be underlined that more complicated models in practice are not acceptable by clinicians.

It was very difficult to calculate the threshold value for $\tau_{2}$ parameter as the class discriminant (burn wound which will spontaneously healed, burn wound which will not spontaneously healed), but definition of the derivative normalized parameter formula (5) - "normalized time constant":

$$
\tau_{2 n_{-} i}=\frac{\tau_{2_{-} i}-\tau_{2_{-} r e f}}{\tau_{2_{-} i}+\tau_{2_{-} r e f}},
$$

where: $\tau_{2} \mathrm{i}$ - the time constant for $\mathrm{i}$-th pixel in the parametric image, $\tau_{2}$ ref - the averaged time constant for unburned tissue chosen in a reference region, makes such discrimination of threshold value possible. The theoretical range of the coefficient value is $[-1,1]$. The value of the normalized thermal time constant used in our work [1] as the discriminator parameter is marked as $\tau_{2 n_{\_} \text {. }}$. The calculated threshold value of the normalized time constant is $\tau_{2 n_{-} \text {treshold }}=0.000113$ and the quality parameters of the method are as: accuracy $-100 \%$, sensitivity $-100 \%$ and specificity $-100 \%$. These results were obtained for several series of in-vivo experiments on domestic pigs.

\section{Results}

As an example of the results one of the clinical case investigations is shown: a burn wound to the abdomen in 19 year old man. Most of the surfaces of the burn had a difficult to determine depth. The patient was treated conservatively. After three weeks, the whole area healed spontaneously, which confirmed the thermographic observation. Fig. 4 shows the ADT parametric images. All area are classified as to be healed spontaneously within 3 weeks basing on normalized thermal time constant parameter.

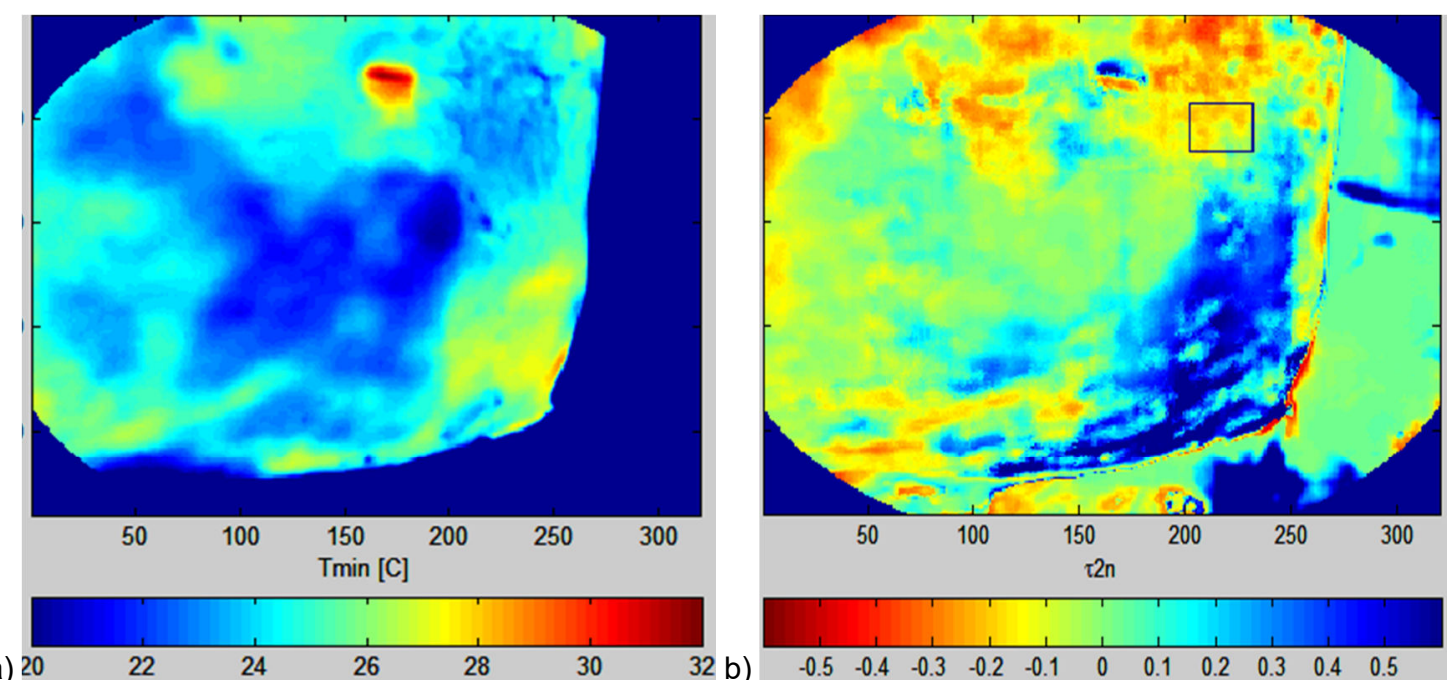

Fig. 4. Clinical case from Fig. 1 - ADT parametric images of the two-exponential thermal model for $\mathrm{CO}_{2} \mathrm{Cooling}$ a) temperature at the moment of cooling termination; b) derivative normalized time constant for the reference region indicated in the rectangle - equation 5

One may interpret the images after training in analysis of ADT images. In Fig. 4 b) the parametric image of the normalized time constant $\tau_{2 n}$ (eq. 5) shows relatively uniform distribution (close to zero value or above zero) what should 
be interpreted as predicted natural healing of the full burn wound. This simple quantitative decision criterion is fully accepted by medical staff.

In Fig. 5 parametric images of TSR polynomial coefficients are shown. Coefficient a0 is proportional to temperature and its interpretation is quite easy. Interpretation of other factors is more complicated and it is hard to say which mechanism plays a greater role. Looking at parametric images, it is very difficult to classify the tissue surface into two groups (classes): unburned (healthy) skin and superficial burn area. Only the image of the coefficient a0 allows such a classification.
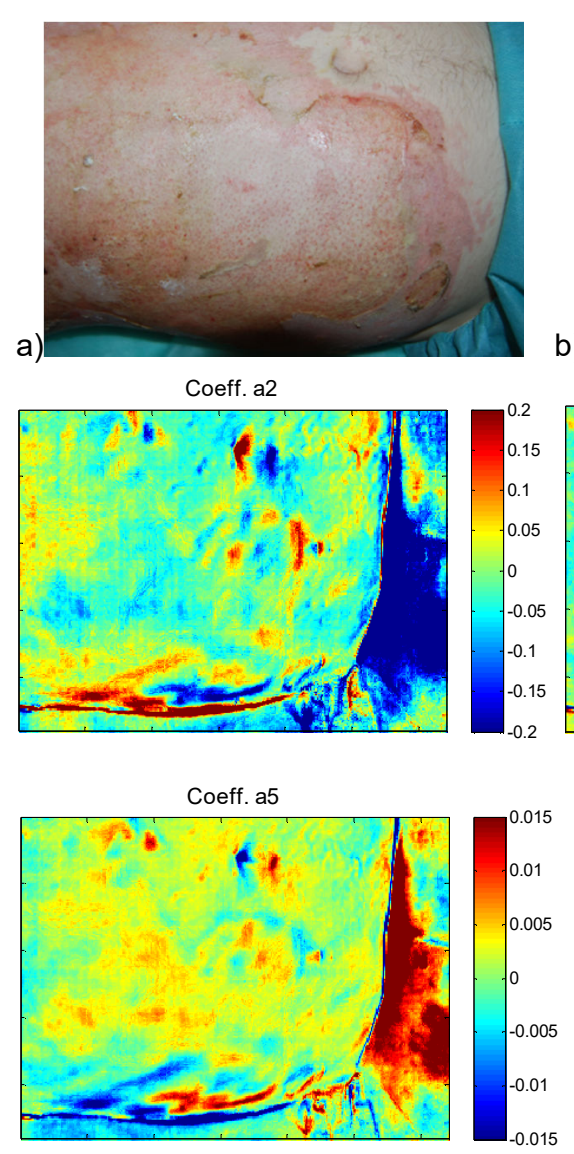

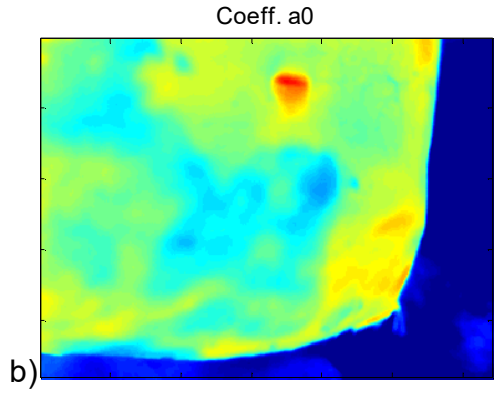

Coeff. a3

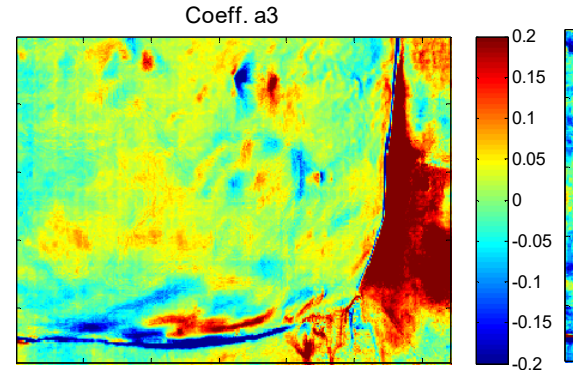

Coeff. a6

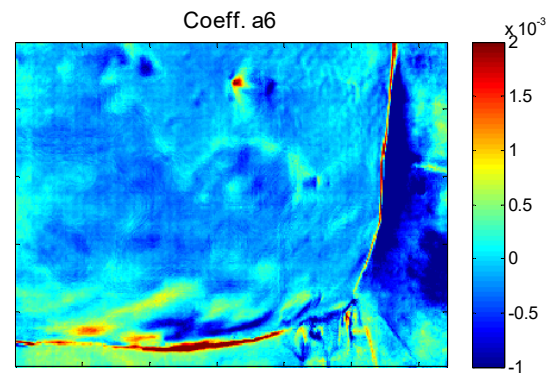

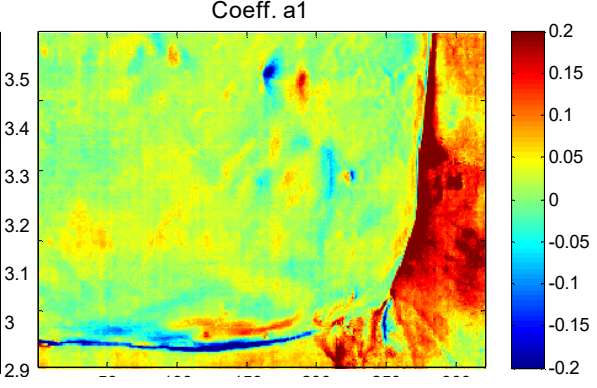

Coeff. a4

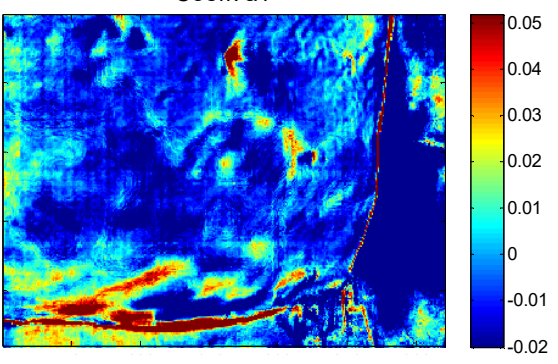

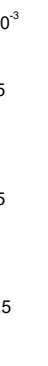

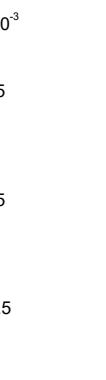

Fig. 5. a) Photograph of the burned abdomen on post burn day 2, b) corresponding TSR images of seven polynomial coefficients

Table 1 summarizes the determined parameters for the exponential model (4) and the TSR polynomial model (2). The determined coefficients for this particular case of the patient do not allow for a simple division of the skin surface into a scalded and non-scorched area.

Table 1. Calculated parameters of compared models

\begin{tabular}{|c|c|c|c|c|c|c|c|}
\hline \multicolumn{8}{|c|}{ ADT - two-exponential model } \\
\hline Parameter & $\mathrm{T}_{\min }\left[{ }^{0} \mathrm{C}\right]$ & $\Delta \mathrm{T}_{1}\left[{ }^{0} \mathrm{C}\right]$ & $\tau_{1}$ & $\Delta \mathrm{T}_{2}\left[{ }^{0} \mathrm{C}\right]$ & $\tau_{2}$ & $\tau_{2 n}$ & \multirow[t]{5}{*}{$\tau_{2-\text { ref }}=\mathrm{AR} 01$} \\
\hline AR01 & 23.9 & 2.3 & 3.79 & 3.1 & 38.08 & 0 & \\
\hline AR02 & 23.6 & 3.1 & 2.55 & 3.4 & 34.72 & -0.046 & \\
\hline AR03 & 21.8 & 3.0 & 4.44 & 4.9 & 54.32 & 0.176 & \\
\hline AR04 & 27.0 & 1.5 & 4.95 & 2,0 & 41.48 & 0.043 & \\
\hline \multicolumn{8}{|c|}{ TSR method $-6^{\text {th }}$ degree polynomial } \\
\hline & $a_{0}$ & $a_{1}$ & $a_{2}$ & $a_{3}$ & $a_{4}$ & $a_{5}$ & $a_{6}$ \\
\hline AR01 & 3.248 & 0.0096 & 0.0074 & 0.0007 & -0.0012 & 0.0003 & $-2 E-9$ \\
\hline AR02 & 3.265 & -0.0022 & 0.0482 & -0.0281 & 0.0069 & -0.0006 & $-3 E-9$ \\
\hline AR03 & 3.156 & 0.0309 & -0.0289 & 0.0376 & -0.0173 & 0.0035 & -0.0003 \\
\hline AR04 & 3.319 & 0.0301 & -0.0462 & 0.0435 & -0.0173 & 0.0032 & -0.0002 \\
\hline
\end{tabular}


In case of ADT method all values of normalized time constant $\left(\tau_{2 n}\right)$ are close to zero as for reference unburned area, only region AR03 has a little bigger value then reference area and will heal spontaneously within three weeks.

Based on collection of images - Fig. 5, it would be difficult for a doctor to decide on how to treat the case. Further work is needed to determine the decision criterion that is easy to apply in clinical practice. We have managed to analyse more cases of burns, as was the case when developing the ATD method using a two-exponential model.

The TSR method has one main advantage - the calculation procedure is quite fast and allows to obtain results almost in real time, compared to the two exponential ADT model. Drawback of this method is a large set of parametric images $a_{i}$, even an extended TSR procedure [8] with the calculation of the $1^{\text {st }}$ and $2^{\text {nd }}$ derivative is unacceptable for medical personnel in clinical settings.

\section{Conclusions}

This overview shows importance of still new in medicine visualization modality called ADT - Active Dynamic Thermography with exponential model and/or TSR methods. Both modalities are implementing each other, therefore analysis and comparison of proper descriptors allows improvements of diagnostics and support multimodality concept of advanced diagnostics in medicine.

The proposed method allows the reduction of parametric images calculation time (the demand for computing power) needed to obtain predictor correlated with the process of burn wound healing comparing to previous proposed ADT method with exponential model. The work continues and as enlarging collections of cases, well-healing wounds and complicated will be complemented by statistical analysis, in order to fully demonstrate the utility of the proposed methods for assessing the healing of burn wound. Probably the classifier based on a combination of a larger number of predictors is more universal classifier, including the various types of complications of burn wounds.

Analysis of both - the TSR as well as the ADT measurement results leads to the conclusion that special care must be taken to secure proper measurement conditions. Important is also process of experiment simulation necessary in reconstruction of a tested structure.

The clinical results of the work are very promising as the developed of versatile, objective instrumentation allows for qualitative and quantitative evaluation of many superficial symptoms of illness, as burns - depth and region of surgical interventions; skin transplants; breast reconstruction surgery control, evaluation of post surgery wound healing and other.

In summary - ST (static thermography, ADT and TSR are different modalities implementing each other. The combined multimodality data are of much higher specificity, accuracy and sensitivity in many medical investigations. Further matching of other medical imaging methods is a great promise for modern medicine. The decreasing costs of IRthermal imaging makes very optimistic prognosis of multimodality approaches in clinical applications.

Acknowledgments Author acknowledge participation in this work of all co-workers from the Department of Biomedical Engineering Gdansk University of Technology, from the Department of Plastic Surgery, Gdansk University of Medicine. This work was financed by several research grants, and partly from GUT Faculty of Electronics, Telecommunications and Informatics statue funds.

\section{REFERENCES}

[1] Renkielska, A., Kaczmarek, M., Nowakowski, A., Grudziński, J., Czapiewski, P., Krajewski, A., Grobelny, I., Active dynamic infrared thermal imaging in burn depth evaluation, Journal of Burn Care and Research, 2014 Sep-Oct; 35(5):e294-303. doi: 10.1097/BCR.0000000000000059.

[2] Kaczmarek M., Nowakowski A., Active IR-Thermal Imaging in Medicine, J Nondestruct Eval (2016), Springer, 35:19, DOI 10.1007/s10921-016-0335-y, 2016.

[3] Shepard S.M., Lhota J.R., Rubadeux B.A., Wang D. and Ahmed T.: Reconstruction and enhancement of active thermographic image sequences. Optical Engineering 2003, vol. 42, 1337-1342.

[4] Vavilov VP, Burleigh DD. Review of pulsed thermal NDT: physical principles, theory and data processing. NDT \& E Int. 2015;73:28-52.

[5] Wei-Min Liu, J. Maivelett, G.J. Kato, J.G. Taylor, Wen-Chin Yang, Yun-Chung Liu, You-Gang Yang, A.M. Gorbach, Reconstruction of Thermographic Signals to Map Perforator Vessels in Humans, Quant Infrared Thermogr J. 2012 Jan 1; 9(2): 10.1080/17686733.2012.737157.

[6] Balageas D.L., Roche JM., Leroy FH., Liu WM., Gorbach A.M., The thermographic signal reconstruction method: a powerful tool for the enhancement of transient thermographic images, Biocybern Biomed Eng. 2015; 35(1): 1-9.

[7] Shepard S. M. "Advances in Pulsed Thermography", Proc. SPIE - The International Society for Optical Engineering, Thermosense XXVIII, Orlando, FL, 2001, Eds. A. E. Rozlosnik and R. B. Dinwiddie, 4360:511-515, 2001.

[8] Balageas D., Roche J., Leroy F., Liu W., Gorbach A., The Thermographic Signal Reconstruction Method: A Powerful Tool for the Enhancement of Transient Thermographic Images, Biocybernetics and Biomedical Engineering, vol. 35, no 1, 2015. 